\section{RMD Open}

Rheumatic \&

Musculoskeletal Diseases

\title{
Efficacy and safety of colchicine in COVID-19: a meta-analysis of randomised controlled trials
}

Kedar Gautambhai Mehta (D) , ${ }^{1}$ Tejas Patel, ${ }^{2}$ Paragkumar D Chavda, ${ }^{1}$ Parvati Patel ${ }^{1}$

To cite: Mehta KG, Patel T, Chavda PD, et al. Efficacy and safety of colchicine in COVID-19: a meta-analysis of randomised controlled trials. RMD Open 2021;7:e001746. doi:10.1136/ rmdopen-2021-001746

- Additional supplemental material is published online only. To view, please visit the journal online (http://dx.doi.org/10. 1136/rmdopen-2021-001746).

Received 26 May 2021 Accepted 13 September 2021

Check for updates

(C) Author(s) (or their employer(s)) 2021. Re-use permitted under CC BY-NC. No commercial re-use. See rights and permissions. Published by BMJ.

${ }^{1}$ Community Medicine, GMERS Medical College Gotri, Vadodara, Gujarat, India

2Pharmacology, AllMS,

Gorakhpur, Uttar Pradesh, India

Correspondence to

Dr Kedar Gautambhai Mehta; kedar_mehta20@yahoo.co.in

\section{ABSTRACT}

Background Colchicine, an anti-inflammatory drug is prescribed nowadays for COVID-19. In this meta-analysis, we evaluated efficacy and safety of colchicine in patients with COVID-19.

Methods We searched databases for randomised controlled studies evaluating efficacy and/or safety of colchicine as compared with supportive care in patients with COVID-19. The efficacy outcomes were mortality, ventilatory support, intensive care unit (ICU) admission and length of hospital stay. The safety outcomes were adverse events, serious adverse events and diarrhoea. A metaanalytical summary was estimated using random effects model through Mantle-Hanzle method. An $\mathrm{I}^{2}$ test was used to assess heterogeneity. The Grades of Recommendation, Assessment, Development and Evaluation (GRADE) approach was used to assess quality of evidence for each outcome.

Results Out of 69 full texts assessed, 6 studies (16148 patients with COVID-19) were included in meta-analysis. Patients receiving colchicine did not show significant reduction in mortality (risk difference, $\mathrm{RD}-0.00(95 \% \mathrm{Cl}$ -0.01 to 0.01 ), $I^{2}=15 \%$ ), ventilatory support (risk ratio, $R R$ $0.67(95 \% \mathrm{Cl} 0.38$ to 1.21$\left.),\left.\right|^{2}=47 \%\right)$, ICU admission (RR 0.49 (95\% Cl 0.19 to 1.25$), \mathrm{I}^{2}=34 \%$ ), length of hospital stay (mean difference: $-1.17(95 \% \mathrm{Cl}-3.02$ to 0.67$)$, $\left.\mathrm{I}^{2}=77 \%\right)$ and serious adverse events (RD $-0.01(95 \%$ $\mathrm{Cl}-0.02$ to 0.00$), \mathrm{I}^{2}=28 \%$ ) than those who received supportive care only. Patients receiving colchicine had higher rates of adverse events (RR 1.58 (95\% Cl 1.07 to 2.33$), I^{2}=81 \%$ ) and diarrhoea (RR 1.93 (95\% Cl 1.62 to 2.29$), I^{2}=0 \%$ ) than supportive care treated patients. The GRADE quality of evidence was moderate for most outcomes.

Conclusion The moderate quality evidence suggests no benefit of addition of colchicine to the standard care regimen in patients with COVID-19.

\section{INTRODUCTION}

SARS-CoV-2, a novel coronavirus, is a singlestranded RNA virus discovered in Wuhan, China in 2019. It has resulted in a devastating pandemic of COVID-19 since 11 March 2020, as declared by WHO. As of 20 May 2021, there are around 164.40 million confirmed

\section{Key messages}

What is already known about this subject?

- Considering the anti-inflammatory role of colchicine, several physicians have tried it for COVID-19 management.

- Previous randomised controlled trials (RCTs) and meta-analyses have concluded that colchicine is effective in reducing mortality among patients with COVID-19.

What does this study add?

- The strength of this meta-analytical summary is inclusion of RCTs.

- Colchicine does not reduce the risk of mortality, need for ventilatory support, intensive care unit admission or length of hospital stay among patients with COVID-19.

How might this impact on clinical practice or further developments?

- There is no additional benefit of adding colchicine to supportive care in management of patients with COVID-19.

COVID-19 cases and 3.40 million deaths due to COVID-19 reported globally. ${ }^{1}$

Acute respiratory distress syndrome (ARDS) is one of the most common cause of mortality in COVID-19. ARDS occurs due to hyperinflammatory response and production of cytokines interleukin-1 (IL-1), IL-6 and tumour necrosis factor (TNF). ${ }^{2}$ The early diagnosis and prompt management of COVID-19 remains an important public health intervention to reduce mortality.

Many clinical trials including drugs such as hydroxychloroquine, azithromycin, remdesivir and lopinavir-ritonavir have not shown promising results for treatment of COVID-19. ${ }^{3-6}$ One of the recent clinical trial findings have concluded that tocilizumab improves survival and other clinical outcomes in severe patients with COVID-19. 
However, until, there is no proven effective treatment for COVID-19.

Colchicine is an anti-inflammatory drug, known to reduce inflammation in many inflammatory diseases viz. gout, familial mediterranean fever and pericarditis. ${ }^{89}$ Its beneficial effect in patients with COVID-19 could be due to following actions. First, the anti-inflammatory properties of colchicine are exhibited through interfering several inflammatory pathways such as reducing neutrophils adhesion, superoxide anions production and release of TNF-alpha (TNF-alpha) ${ }^{10}$ Second, it is a microtubule inhibitor leading to increased intracellular $\mathrm{pH}$ and inhibits NALP3 inflammasome activation. ${ }^{11}$ Third, it has ability to interfere with NLRP3 inflammatory protein complex leading to inhibition of IL production viz. IL-1, IL-6 and IL-18 which may be responsible for reduction of cytokine storm in patients with COVID-19. ${ }^{12}$ Based on the above-mentioned inflammatory pathophysiology of COVID-19, colchicine has been explored for treatment of COVID-19.

Few systematic reviews conducted earlier on the safety and efficacy of colchicine in COVID-19 management included observational studies in their analysis pool. ${ }^{13-16}$ Now, randomised controlled trials (RCTs) are available evaluating the use of colchicine in patients with COVID19. Hence, this meta-analysis was conducted with an objective to determine the efficacy and safety outcomes in patients with COVID-19 treated with colchicine.

\section{MATERIAL AND METHODS}

\section{Search strategy and study identification}

Two investigators (KGM and TP) independently and systematically searched the data sources PubMed and Google Scholar. The search terms used were: (Colchicine) AND (COVID-19 OR SARS-CoV-2). Additional articles and preprint versions were searched on medrxiv. org, biorxiv.org and and trial registries (ClinicalTrials. gov, clinicaltrialsregister.eu, srctn.com). The last search was performed on 20 July 2021. There were no restrictions due to language or time period of publications to include the studies. Two reviewers (KGM and TP) independently assessed the titles, abstracts and full articles (if required) and decided the eligibility of retrieved articles. Any disagreements for the study selection (inclusion/ exclusion) were resolved by discussion, consensus among the assessing authors and consultation with third author (PDC).

\section{Study selection criteria}

We included all RCTs (open labelled or blinded) investigating efficacy and/or safety of colchicine with any other intervention (supportive care, standard care, placebo or any other treatment modalities) in patients with COVID19. We excluded observational studies (cross-sectional, cohort or case-control), non-interventional studies, in vitro or cell culture, studies conducted on animals, nonSARS-CoV-2 or non-COVID-19 studies, studies having less than 10 participants in the treatment arms, non-research articles (eg, review articles, meta-analysis) and duplicate publications.

\section{Study population}

The study population consisted of any age group patients with any severity of laboratory-confirmed COVID- 19 receiving treatment in any setting (inpatient or outpatient basis) as a participant of RCT.

\section{Types of interventions}

Use of colchicine was considered irrespective of dose and duration of therapy. The interventions as an add on or concomitant to colchicine treatment arm were analysed separately.

\section{Types of comparators}

All comparators (supportive care, placebo or any treatment modalities) were considered as the control arm.

\section{Quality analysis}

Two investigators (KGM and TP) critically evaluated the methodological quality of the included studies as per revised Cochrane 'risk of bias assessment tool for the RCTs (ROB-II).${ }^{17}$ The following five domains were assessed: bias arising from the randomisation process, bias due to deviations from intended interventions, bias due to missing outcome data, bias in outcome measurement and bias in selection of reported results. Each domain was categorised into 'low', 'high' or having 'some concerns' as per ROB-II tool. ${ }^{17}$ Any disagreements were resolved by discussion and consensus among the authors.

\section{Data collection and extraction}

The following data were extracted in a Microsoft Excel sheet: first author, publication year, study design, study site, study population in treatment arms (demography, disease severity), interventions (dosage, duration and route of administration), supportive care, efficacy data (mortality, need of ventilatory support, need of intensive care unit (ICU) admission, length of hospital stay) and safety data (adverse events, serious adverse events (SAEs), diarrhoea-individual adverse event). In case of missing outcome data, the corresponding authors were contacted through mail to provide the data and it was included in the analysis as received from the investigator team. The data were extracted by first author (KGM) and cross-checked by second author (TP) to ensure the accuracy of data extraction.

\section{Efficacy outcomes}

The following parameters were defined as efficacy outcomes: mortality, need of ventilatory support, need of ICU admission and length of hospital stay among patients receiving colchicine and control interventions (standard care). In case of multiple timepoints estimation, data at the end of the study period were taken into analysis. The intention to treat population was used to estimate the efficacy outcomes. 


\section{Safety outcome}

The following safety outcomes were assessed using safety populations: adverse events, gastrointestinal adverse events (diarrhoea) and SAEs between colchicine and control interventions at the end of the study period. All patients who received colchicine and control interventions irrespective of their per-protocol doses and durations were considered as a safety population.

\section{Measure of treatment effects and data synthesis}

All outcomes were the dichotomous except length of hospital stay (continuous outcome). The dichotomous outcomes were summarised as a risk difference (RD) or a risk ratio (RR) with $95 \% \mathrm{CI}$, while continuous outcome was summarised as a mean difference (MD) $(95 \% \mathrm{CI})$. The meta-analytical summary was pooled through the fixed effect or random model using the Mantle-Hanzle method. The random effect model was used in case of clinically and statistically heterogenous data. The statistical heterogeneity was assessed using $I^{2}$ test. The 'funnel plot' was used to assess publication bias.

\section{Reporting bias and sensitivity analysis}

The publication bias was assessed through 'funnel plot' of effect estimates and precision of all outcomes. The sensitivity analyses of all outcomes were performed to assess influence of study quality and follow-up duration of studies. Each outcome was estimated by excluding studies showing 'some concern' or 'high' risk of bias in case of sensitivity analysis as per study quality. Each outcome was also estimated by excluding studies having $\leq 14$ days of follow-up in sensitivity analysis as per follow-up duration of studies

\section{Quality of evidence for the meta-analytical summary}

The quality of the evidence for each outcome was rated as per the Grades of Recommendation, Assessment, Development and Evaluation (GRADE) approach using following key elements: study limitations, inconsistency, indirectness of evidence, imprecision and publication bias. The evidence was rated into four categories: high, moderate, low and very low quality. ${ }^{18}$

The meta-analysis was performed through 'Review manager software V.5.3'.
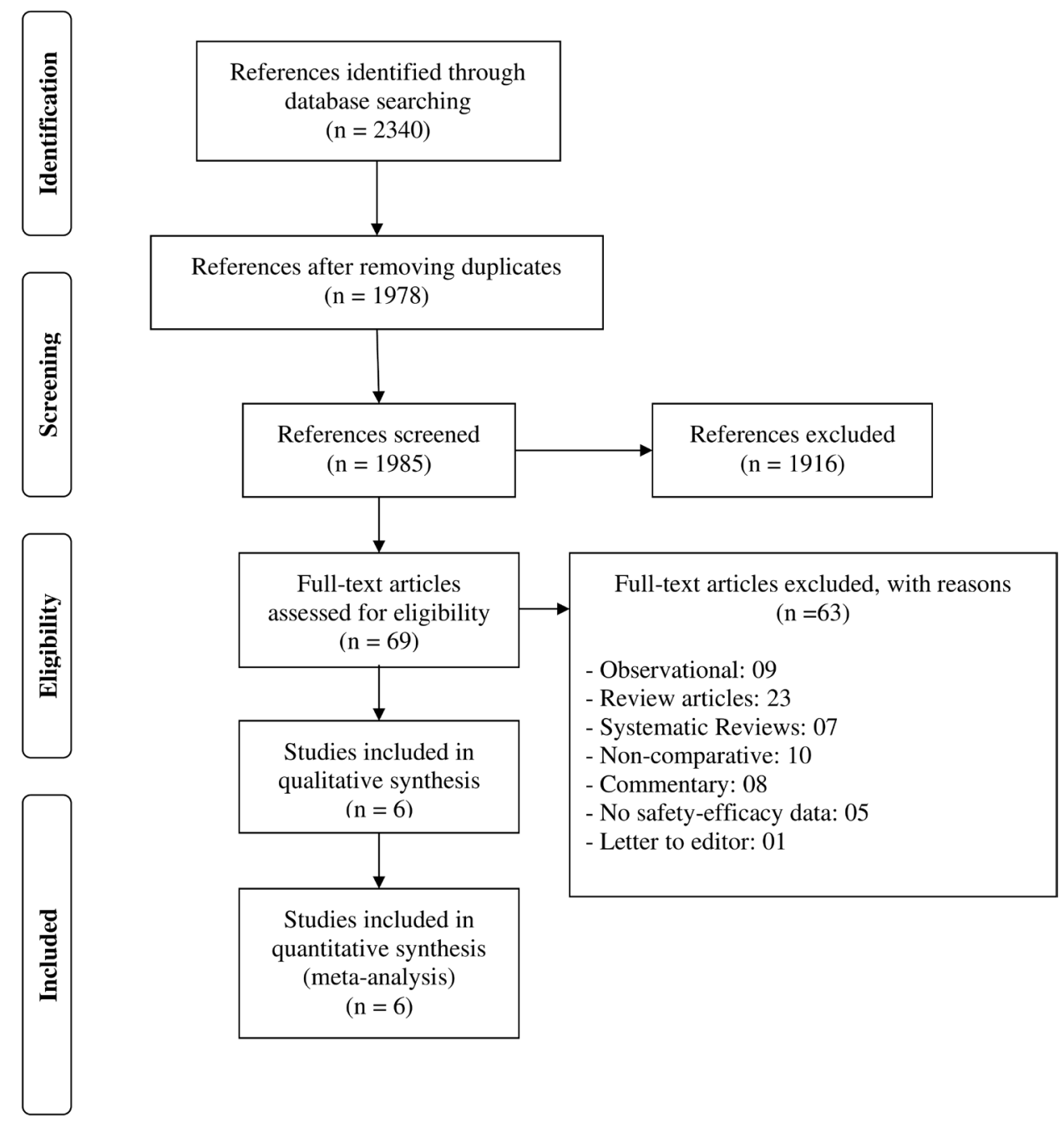

Figure 1 PRISMA flow diagram showing selection of studies. PRISMA, Preferred Reporting Items for Systematic Reviews and Meta-Analyses. 


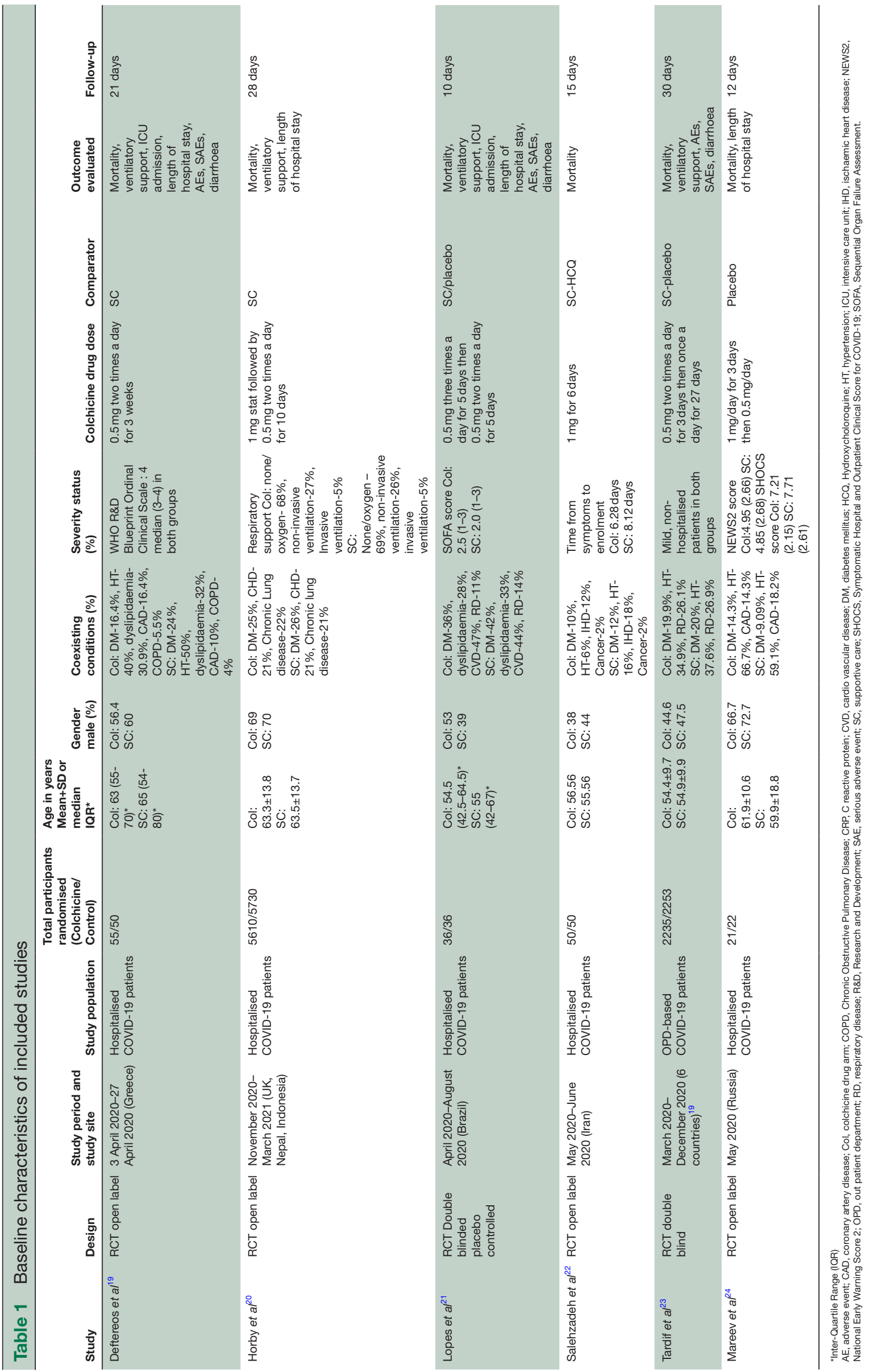


Figure 2 Quality assessment of included studies as per risk of bias assessment tool - II.

\section{RESULTS}

\section{Trial characteristics}

The database search strategy retrieved 1985 bibliographic records. Total 69 full-text records were assessed. Six randomised controlled studies were included in the final analysis (figure 1), which comprised 16148 patients with COVID-19 (8007 received colchicine drug and 8141 received supportive care). The control interventions were supportive care in all studies. The general characteristics of all included randomised studies are presented in table $1 .{ }^{19-24}$ Four randomised studies had used open labelled ${ }^{192022} 24$ and two used double-blind design. ${ }^{21} 23$ All, except one study, were conducted on the hospitalised patients with COVID-19. Tardif et al had conducted study in the outpatient setting. ${ }^{23}$ The participants in treatment arms were comparable for the median or mean age groups, comorbid conditions viz. diabetes mellitus, cardiovascular diseases and respiratory disease in all included studies. All studies had used varying definitions of COVID-19 severity. The participants in treatment arms were comparable for the WHO clinical scale, ${ }^{19}$ respiratory support, ${ }^{20}$ Sequential Organ Failure Assessment Score, ${ }^{21}$ National Early Warning Score 2, ${ }^{24}$ symptomatic hospital and outpatient clinical score for COVID-19, ${ }^{24}$ time from symptom to enrolment. ${ }^{22}$

\section{Quality assessment of included studies}

The methodological quality assessment for individual trials is summarised in figure 2. Four included studies were considered as having a 'low' risk of bias. Mareev $e t$ $a l^{24}$ were considered having a 'high risk' of bias randomisation process and deviations from intended interventions. Salehzadeh et $a l^{22}$ were considered to have 'some concerns' due to missing outcome data as per ROB-II tool.

\section{Efficacy outcomes}

Mortality

Six studies (16148 participants) contributed to mortality data analyses. No significant difference in risk reduction of mortality was observed between patients treated with colchicine and supportive care (RD $-0.00 \quad(95 \%$ CI -0.01 to 0.01 )) (figure 3 ). An $\mathrm{I}^{2}$ of $15 \%$ suggested a low heterogeneity. The funnel plot was asymmetrical on visual inspection (online supplemental figure 1). A sensitivity analysis based on risk of bias assessment (excluding studies by Salehzadeh $e t a l^{22}$ and Mareev $e t$ $\left.a l^{24}\right)$ and follow-up duration of studies (excluding studies conducted by Lopes $e t a l^{21}$ and Mareev $e t a l^{24}$ ) did not affect mortality outcome (online supplemental figures 2 and 3) The GRADE approach suggested a moderate quality of evidence for the mortality outcome (table 2).

\section{Ventilatory support}

Meta-analytical summary based on 5 studies ( 15519 participants) demonstrated no significant difference in requirement of ventilatory support between patients treated with colchicine and supportive care (RR 0.67 (95\% CI 0.38 to 1.21 ); $\mathrm{I}^{2}=47 \%$; GRADE approach evidence: moderate quality). A sensitivity analysis based on risk of bias assessment and follow-up duration of studies did not affect the outcome (online supplemental figures 2 and 3 ).

\section{ICU admission}

Meta-analytical summary based on 3 studies (220 participants) demonstrated no significant reduction in ICU admission in patients treated with colchicine than supportive care (RR 0.49 (95\% CI 0.19 to 1.25 ); $\mathrm{I}^{2}=34 \%$ ). The GRADE approach suggested a moderate quality of evidence for the ICU admission (table 2). A sensitivity analysis based on risk of bias assessment and follow-up 


\section{A. Mortality}

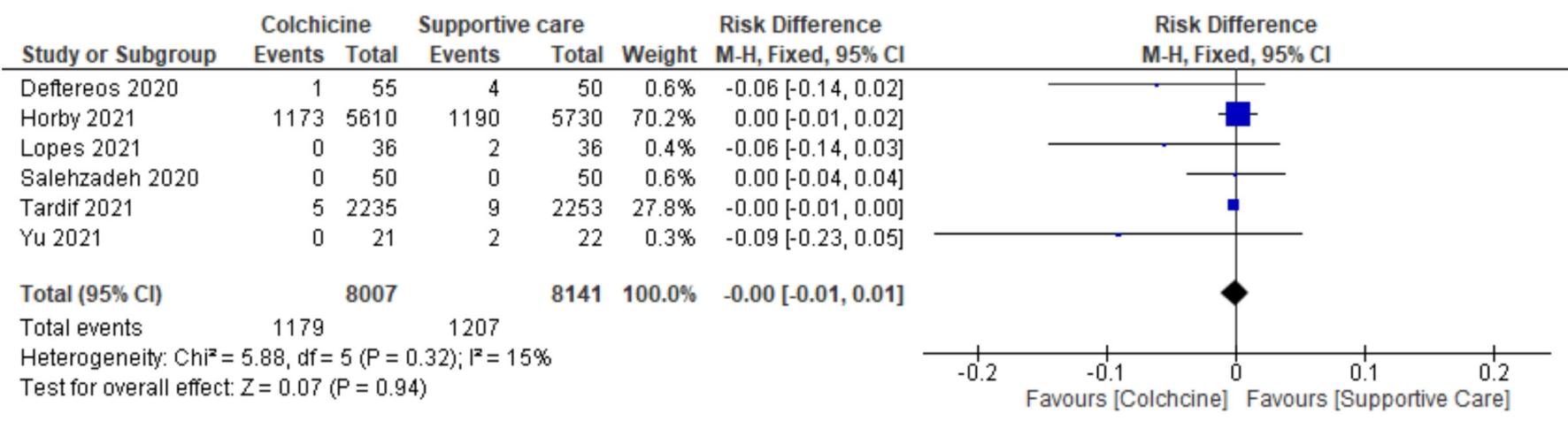

\section{B. Ventilatory support}

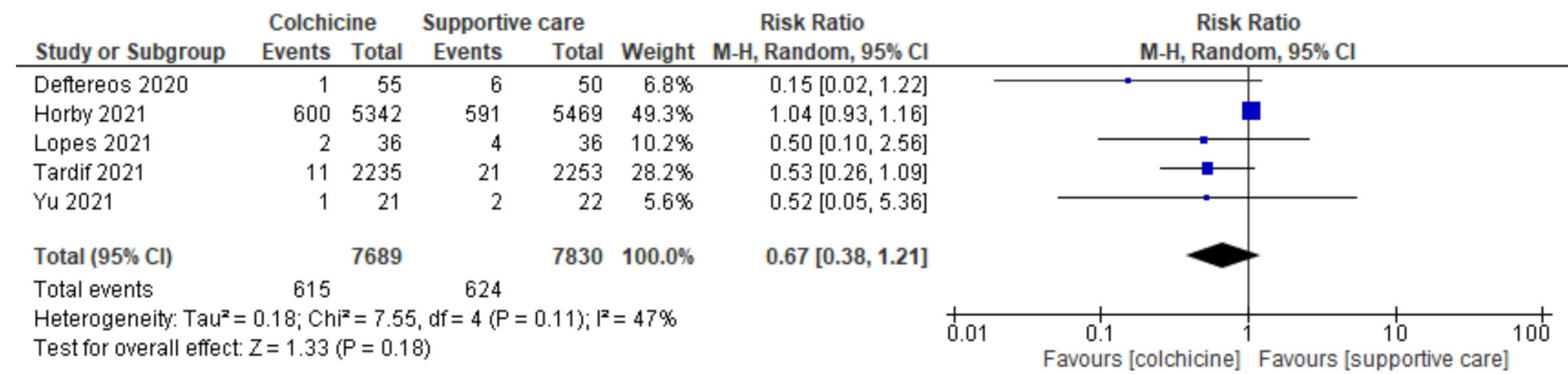

\section{ICU admission}

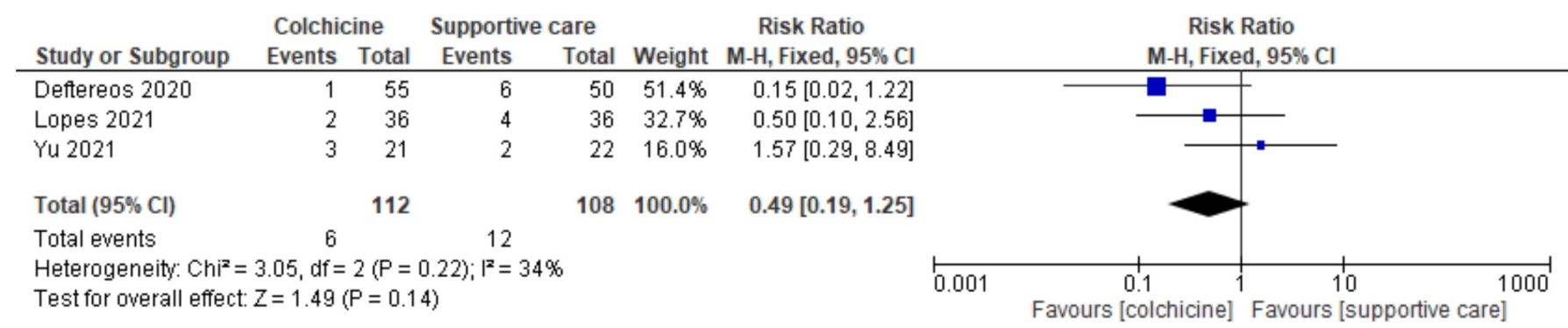

\section{Length of hospital stay}

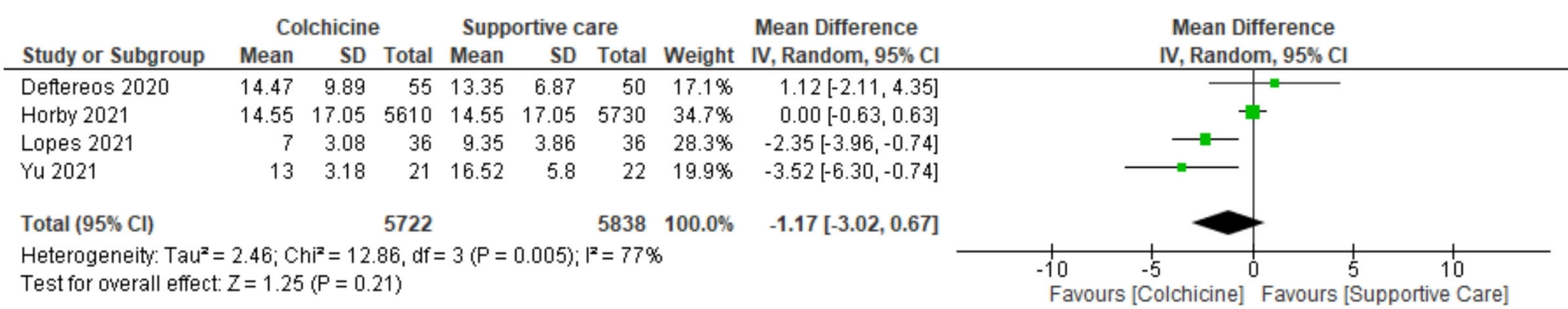

Figure 3 Meta-analytical summary of efficacy outcomes. ICU, intensive care unit; IV, inverse variance; M-H, Mantel-Haenszel. 


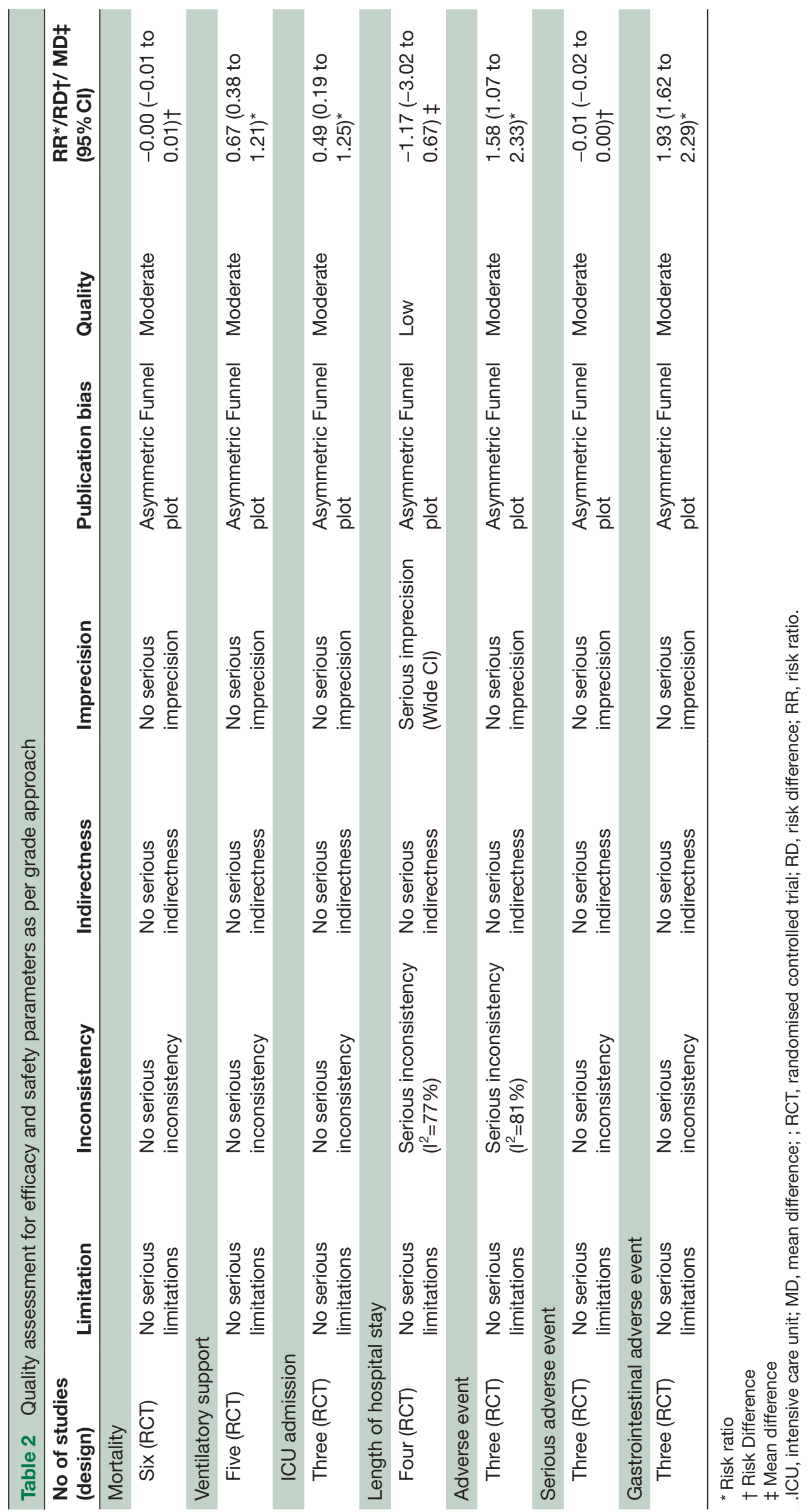




\section{A. Adverse Events}

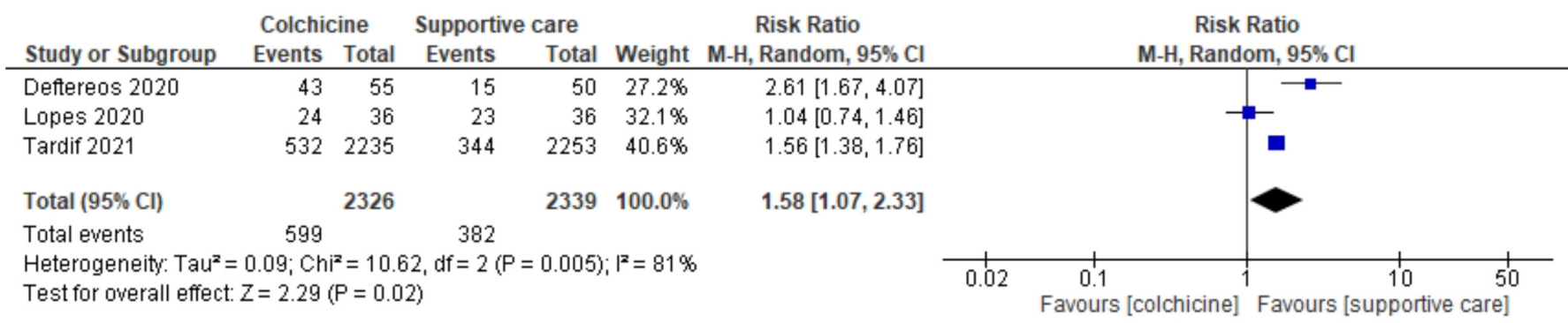

\section{B. Serious adverse event}

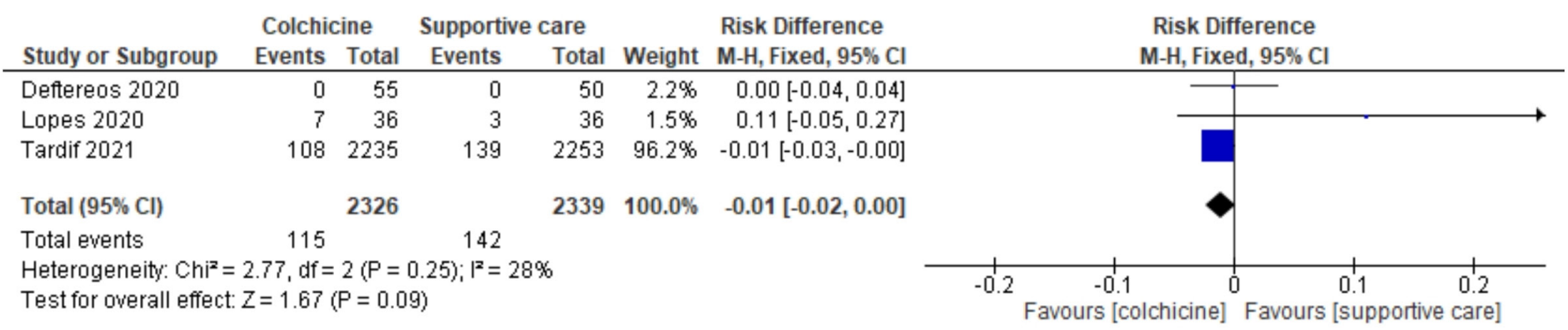

\section{Diarrhoea - Adverse event}

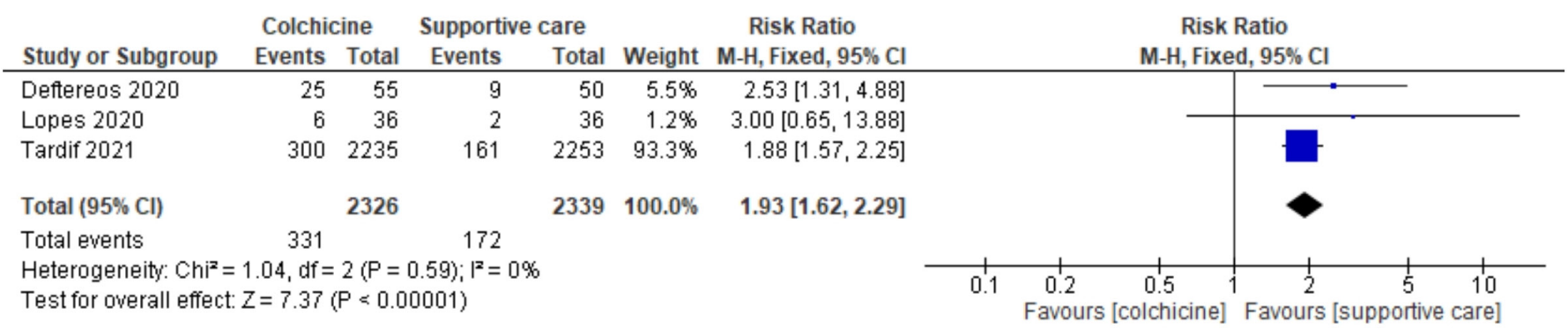

Figure 4 Meta-analytical summary of safety outcomes. M-H, Mantel-Haenszel.

duration of studies did not affect this outcome (online supplemental figures 2 and 3 ).

\section{Length of hospital stay}

Meta-analytical summary based on four studies (11560 participants) demonstrated that patients who received colchicine had no difference in hospital stay (MD -1.17 (95\% CI -3.02 to 0.67); GRADE approach evidence: low quality) as compared with those who received supportive care (figure 3). An $\mathrm{I}^{2}$ of $77 \%$ suggested a significant between-trial heterogeneity. A sensitivity analysis based on risk of bias assessment and follow-up duration of studies did not affect this outcome (online supplemental figures 2 and 3 ).

\section{Safety outcomes}

Three low risk of bias studies (4665 participants) contributed to adverse events, SAEs and gastrointestinal adverse events (diarrhoea) data analyses. As shown in figure 4, patients treated with colchicine had higher risk of adverse events (RR 1.58 (95\% CI 1.07 to 2.33 ); $\mathrm{I}^{2}=81 \%$ ) and diarrhoea (RR 1.93 (95\% CI 1.62 to 2.29 ); $\mathrm{I}^{2}=0 \%$ ) than those treated with supportive care. We found no difference in risk of SAEs between patients treated with colchicine and supportive care (RD -0.01 (95\% CI -0.02 to 0.00$) ; I^{2}=28 \%$ ). GRADE analysis suggested moderate quality of evidence for the safety outcomes. A sensitivity analysis based on follow-up duration of studies did not affect safety outcomes (online supplemental figure 3).

\section{DISCUSSION}

Our findings suggest no definitive clinical benefit of addition of colchicine in the treatment of COVID-19. Colchicine was not found to be beneficial in reducing mortality, need of ventilatory support or need of ICU admission in patients with COVID-19. A similar trend was observed in the sensitivity analysis. High risk of adverse events was observed in patients treated with colchicine as compared 
with control interventions. There was no difference in occurrence of SAE.

Colchicine is not beneficial in reducing risk of mortality in patients with COVID-19. However, earlier meta-analysis studies done by Chiu et $a l^{13}$, Vrachatis et $a l^{16}$ and Hariyanto $e t a l^{14}$ concluded that colchicine may reduce the mortality risk among patients with COVID19. A living systematic review and network meta-analysis conducted by Siemieniuk et $a l^{25}$ concluded that colchicine may reduce mortality and mechanical ventilation in non-severe patients with COVID-19. The major reason for contradictory finding could be the inclusion of mainly observational studies and RCTs in their metaanalysis summary model while we have included only RCTs in this meta-analysis. The other reason being all included studies had some concern for risk of bias in all previous meta-analyses. Moreover, our study has included the findings of recent large-scale Randomised Evaluation of COVID-19 therapy (RECOVERY) trial, which has contributed to the estimates in our study and concluded that colchicine has no effect on mortality and mechanical ventilation.

Although colchicine has proved effective for various chronic inflammatory conditions, its non-beneficial effect in COVID-19 has been linked to its effect on intracellular $\mathrm{pH}$. The effect of colchicine on intracellular $\mathrm{pH}$ is dynamic with a net result being that it fails to raise intracellular $\mathrm{pH}$ to a level that prevents virus from binding to ACE2. ${ }^{26}$ Hence, it is argued that the viral load reduction effect of colchicine may be weak. Further, colchicine is earlier reported to decrease secretion of surfactants at high therapeutic doses which potentially adds to increased risk of ARDS and multiorgan failure in COVID-19. ${ }^{27}$

A meta-analysis on safety profile of colchicine conducted by Stewart $e t a l^{28}$ during pre-COVID-19 time reported that colchicine is a well-tolerated drug and has a good safety profile except higher chance of diarrhoea adverse event. Similar finding was observed in our meta-analysis pooled model among patients with COVID-19. So, considering the risk-benefit approach, colchicine offers no additional benefit of reducing mortality, ICU admission or need of ventilatory support among Cpatients with COVID-19 and adds to the risk of gastrointestinal adverse effects. Although, colchicine was thought to be a cost-effective option as compared with other antivirals and medications such as remdesivir and tocilizumab; but the results do not show any benefit.

This study has several strengths. First, we have included only RCTs in our meta-analysis summary. Second, our study includes summary of 16148 patients with COVID-19 which indicates higher statistical power as compared with previous meta-analyses. Third, we have used standard ROB-II and GRADE approach to ensure data quality for the inclusion of studies in our model.

This study has few limitations. We have restricted our search to free databases only; so, we have not searched studies in EMBASE and CINAHL databases. Our findings on colchicine should be interpreted cautiously due to the inclusion of open labelled randomised clinical trials. The analysis of efficacy and safety outcomes are based on a small number of RCTs in control interventions.

In conclusion, colchicine does not reduce mortality, need for ventilatory support and ICU admission or length of hospital stay in patients with COVID-19. Patients with COVID-19 receiving colchicine are at two times higher risk of developing diarrhoea compared with standard care. The risk of SAEs is same with colchicine as standard care.

\section{Twitter Tejas Patel @drtejas_patel}

Contributors All authors were involved in conception and design of the metaanalysis; KGM and TP conducted the literature search and extracted the data; all authors have analysed and interpreted the data; KGM wrote the first draft of the manuscript and all authors gave significant inputs and approved the final version of the manuscript. KGM is responsible for the overall content as the guarantor.

Funding The authors have not declared a specific grant for this research from any funding agency in the public, commercial or not-for-profit sectors.

Competing interests None declared.

Patient consent for publication Not applicable.

Provenance and peer review Not commissioned; externally peer reviewed.

Data availability statement All data relevant to the study are included in the article or uploaded as online supplemental information.

Open access This is an open access article distributed in accordance with the Creative Commons Attribution Non Commercial (CC BY-NC 4.0) license, which permits others to distribute, remix, adapt, build upon this work non-commercially, and license their derivative works on different terms, provided the original work is properly cited, appropriate credit is given, any changes made indicated, and the use is non-commercial. See: http://creativecommons.org/licenses/by-nc/4.0/.

ORCID iD

Kedar Gautambhai Mehta http://orcid.org/0000-0003-4374-9495

\section{REFERENCES}

1 World Health Organisation. Coronavirus disease (COVID-19) situation reports, 2021. Available: https://www.who.int/emergencies/ diseases/novel-coronavirus-2019/situation-reports

2 Yuki K, Fujiogi M, Koutsogiannaki S. COVID-19 pathophysiology: a review. Clin Immunol 2020;215:108427.

3 WHO Solidarity Trial Consortium, Pan H, Peto R, et al. Repurposed Antiviral Drugs for Covid-19 - Interim WHO Solidarity Trial Results. N Engl J Med 2021;384:497-511.

4 RECOVERY Collaborative Group. Azithromycin in patients admitted to hospital with COVID-19 (recovery): a randomised, controlled, open-label, platform trial. Lancet 2021;397:605-12.

5 RECOVERY Collaborative Group, Horby P, Mafham M, et al. Effect of hydroxychloroquine in hospitalized patients with Covid-19. N Engl J Med 2020;383:2030-40.

6 RECOVERY Collaborative Group. Lopinavir-ritonavir in patients admitted to hospital with COVID-19 (recovery): a randomised, controlled, open-label, platform trial. Lancet 2020;396:1345-52.

7 RECOVERY Collaborative Group. Tocilizumab in patients admitted to hospital with COVID-19 (recovery): a randomised, controlled, openlabel, platform trial. Lancet 2021;397:1637-45.

8 Khanna D, Khanna PP, Fitzgerald JD, et al. 2012 American College of rheumatology guidelines for management of gout. Part 2: therapy and antiinflammatory prophylaxis of acute gouty arthritis. Arthritis Care Res 2012;64:1447-61.

9 Ozen S, Demirkaya E, Erer B, et al. EULAR recommendations for the management of familial Mediterranean fever. Ann Rheum Dis 2016;75:644-51.

10 Schlesinger N, Firestein BL, Brunetti L. Colchicine in COVID-19: an old drug, new use. Curr Pharmacol Rep 2020:137-45.

11 Leung YY, Yao Hui LL, Kraus VB. Colchicine-update on mechanisms of action and therapeutic uses. Semin Arthritis Rheum 2015;45:341-50.

12 Vitiello A, Ferrara F. Colchicine and SARS-CoV-2: management of the hyperinflammatory state. Respir Med 2021;178:106322. 
13 Chiu L, Chow R, Chiu N. Colchicine use in patients with COVID-19: a systematic review and meta-analysis. medRxiv 2021.

14 Hariyanto TI, Halim DA, Jodhinata C, et al. Colchicine treatment can improve outcomes of coronavirus disease 2019 (COVID-19): a systematic review and meta-analysis. Clin Exp Pharmacol Physiol 2021:48:1-8.

15 Salah HM, Mehta JL. Meta-Analysis of the effect of colchicine on mortality and mechanical ventilation in COVID-19. Am J Cardiol 2021;145:170-2.

16 Vrachatis DA, Giannopoulos GV, Giotaki SG, et al. Impact of colchicine on mortality in patients with COVID-19: a meta-analysis. Hell J Cardiol 2021;314.

17 Sterne JAC, Savović J, Page MJ, et al. Rob 2: a revised tool for assessing risk of bias in randomised trials. BMJ 2019;366:14898.

18 Guyatt GH, Oxman AD, Vist GE, et al. Grade: an emerging consensus on rating quality of evidence and strength of recommendations. BMJ 2008;336:924-6.

19 Deftereos SG, Giannopoulos G, Vrachatis DA, et al. Effect of colchicine vs standard care on cardiac and inflammatory biomarkers and clinical outcomes in patients hospitalized with coronavirus disease 2019: the GRECCO-19 randomized clinical trial. JAMA Netw Open 2020;3:e2013136.

20 Horby PW, Campbell M, Spata E, et al. Colchicine in patients admitted to hospital with 4 COVID-19 (recovery): a randomised, controlled, 5 open-label, platform trial. medRxiv 2021:1-30.
21 Lopes MI, Bonjorno LP, Giannini MC, et al. Beneficial effects of colchicine for moderate to severe COVID-19: a randomised, double-blinded, placebo-controlled clinical trial. RMD Open 2021;7:e001455-8.

22 Salehzadeh F, Pourfarzi F, Ataei S. The impact of colchicine on the COVID-19 patients; a clinical trial study. Res Sq 2020:1-11.

23 Tardif J-C, Bouabdallaoui N, L'Allier PL, et al. Colchicine for community-treated patients with COVID-19 (COLCORONA): a phase 3, randomised, double-blinded, adaptive, placebo-controlled, multicentre trial. Lancet Respir Med 2021;9:924-32.

24 Mareev VY, Orlova YA, Plisyk AG, et al. Proactive anti-inflammatory therapy with colchicine in the treatment of advanced stages of new coronavirus infection. The first results of the COLORIT study. Kardiologiia 2021;61:15-27.

25 Siemieniuk RAC, Bartoszko JJ, Ge L, et al. Drug treatments for covid-19: living systematic review and network meta-analysis. BMJ 2020;45:m2980.

26 Cumhur Cure M, Kucuk A, Cure E. Colchicine may not be effective in COVID-19 infection; it may even be harmful? Clin Rheumatol 2020;39:2101-2.

27 Maurizi M, Delorme N, Laprévote-Heully MC, et al. [Acute respiratory distress syndrome in adults in colchicine poisoning]. Ann Fr Anesth Reanim 1986;5:530-2.

28 Stewart S, Yang KCK, Atkins K, et al. Adverse events during oral colchicine use: a systematic review and meta-analysis of randomised controlled trials. Arthritis Res Ther 2020;22:28. 